\title{
Efficiency to Discovery Transgenic Loci in GM Rice Using Next Generation Sequencing Whole Genome Re-sequencing
}

\author{
Doori Park ${ }^{1}$, Dongin Kim² ${ }^{2}$, Green Jang ${ }^{2}$, Jongsung $\mathrm{Lim}^{2}$, Yun-Ji Shin ${ }^{3}$, Jina Kim³ ${ }^{3}$ Mi-Seong Seo ${ }^{3}$, \\ Su-Hyun Park ${ }^{4}$, Ju-Kon Kim ${ }^{4}$, Tae-Ho Kwon ${ }^{3 *}$, Ik-Young Choi ${ }^{1 * *}$ \\ ${ }^{1}$ Institute of Green Bio Science and Technology, Seoul National University, Pyeongchang 25354, Korea, \\ ${ }^{2}$ National Instrumentation Center for Environmental Management, College of Agriculture and Life Sciences, Seoul National \\ University, Seoul 08826, Korea, \\ ${ }^{3}$ Natural Bio-Materials Inc., Wanju 55322, Korea, ${ }^{4}$ Crop Biotech Institute, Green-Bio Science and Technology, Seoul National \\ University, Pyeongchang 25354, Korea
}

\begin{abstract}
Molecular characterization technology in genetically modified organisms, in addition to how transgenic biotechnologies are developed now require full transparency to assess the risk to living modified and non-modified organisms. Next generation sequencing (NGS) methodology is suggested as an effective means in genome characterization and detection of transgenic insertion locations. In the present study, we applied NGS to insert transgenic loci, specifically the epidermal growth factor (EGF) in genetically modified rice cells. A total of $29.3 \mathrm{~Gb}(\sim 72 \times$ coverage) was sequenced with a $2 \times 150$ bp paired end method by Illumina HiSeq2500, which was consecutively mapped to the rice genome and T-vector sequence. The compatible pairs of reads were successfully mapped to 10 loci on the rice chromosome and vector sequences were validated to the insertion location by polymerase chain reaction (PCR) amplification. The EGF transgenic site was confirmed only on chromosome 4 by PCR. Results of this study demonstrated the success of NGS data to characterize the rice genome. Bioinformatics analyses must be developed in association with NGS data to identify highly accurate transgenic sites.
\end{abstract}

Keywords: genetically modified organisms, next generation sequencing (NGS) T-DNA, rice, risk assessment

\section{Introduction}

Genetic engineering technology is widely used in the agricultural and plant biotechnology fields, ranging from the food and feed industries to bio-pharmaceuticals and cosmetics $[1,2]$. The history of genetically modified (GM) technology began with the discovery of plasmid DNA, where the plasmid could be transferred from one cell to another genome [3]. Scientists subsequently applied the basic plasmid vector system principle and developed recombinant DNA technology to create genetically engineered organisms. Today, GM techniques have been applied to various research fields, including crop sciences, drug manufacturing, and animal husbandry.
The development of transgenic biotechnologies over the last 20 years has led to safety concerns regarding genetically modified organisms (GMOs), particularly in food crops and new pharmaceuticals, which are the most controversial issues. Safety concerns regarding GMOs have resulted in research, debates, and ongoing public unease. Therefore, the European Union (EU) and National Institutes of Health $(\mathrm{NIH})$ in the United States proposed an authorization process in commercial GMO use; however, public apprehension for transgenic techniques remains uncertain and controversial [4-8].

Generally, molecular characterization and identification of GMOs are performed using Southern blots and polymerase chain reaction (PCR) based detection followed by conventional sequencing methods [7]. However, these appro-

Received July 25, 2015; Revised August 24, 2015; Accepted August 24, 2015

*Corresponding author: Tel: +82-63-223-4555, Fax: +82-63-223-4550, E-mail: thkwon@jbnu.ac.kr

**Corresponding author: Tel: +82-33-339-5657, Fax: +82-33-339-5753, E-mail: choii@snu.ac.kr

Copyright $\odot 2015$ by the Korea Genome Organization

(c) It is identical to the Creative Commons Attribution Non-Commercial License (http://creativecommons.org/licenses/by-nc/4.0/). 
aches are limited to evaluate whether the host genome has unintended sequence substitutions and indels [9]. Moreover, if sufficient genomic information is not available for the chosen comparative model species, it is difficult to detect the correct transgenic insert site location or sequence contamination of vector DNA $[9,10]$.

Recent publications of GMO molecular characterizations reported the use of next generation sequencing (NGS) approaches as an effective means to detect the precise transgenic insert location $[9,11,12]$. High-throughput DNA sequencing technologies and bioinformatics can be coupled with NGS to offer new possibilities in drawing genetic maps with feasible costs. For these reasons, researchers have tested new approaches in the molecular characterization of GMOs using NGS technologies [9, 10, 12].

Here, we examined transgenic insertion sites using paired-end whole genome re-sequencing data following Yang et al. with modifications [9]. Human epidermal growth factor (EGF) was inserted into GM rice cells, which could produce EGF safety without endotoxin derived from bacteria and was used as material for this study. Deep sequencing was performed with the Illumina Hiseq2500 platforms (Illumina Inc., San Diego, CA, USA). In this pilot study, we demonstrated the potential of NGS for examination of transgenic insertion loci and discuss some technical bottlenecks of this new method.

\section{Methods}

\section{GM rice samples}

The GM rice event PJKS131-2 was transformed with the EGF inserted pJKS131 vector, produced by Natural BioMaterials Inc. (Jeonju, Korea). Taxonomically, the event PJKS131-2 was derived from Oryza sativa L. cv. Dongjin. The T-vector was transformed with rice callus as described by Chan et al. [13]. Transgenic rice calli were incubated with
$50 \mathrm{mg} /$ L of hygromycin B antibiotic (A.G. Scientific Inc., San Diego, CA, USA) for selection. The GM rice callus samples were subjected to NGS and further validated by PCR amplification.

\section{DNA extraction and whole genome shotgun library and sequencing}

The calli of GM rice event PJKS131-2 were collected and stored at $-80^{\circ} \mathrm{C}$. Total genomic DNA was extracted using the CTAB method in liquid nitrogen. Genomic DNA quality was evaluated by $0.5 \%$ agarose gel electrophoresis. Following the quality check, genomic DNA was sheared with average $500 \mathrm{bp}$ fragment sizes. Truseq DNA PCR free Library Preparation Kit (Illumina Inc.) was used to construct the DNA library according to the manufacturer's protocol. The quality of constructed DNA libraries was confirmed by the LabChip GX system (PerkinElmer, Waltham, MA, USA). DNA libraries were sequenced with 150-bp paired-end sequencing using Illumina Hiseq2500.

\section{Transgenic insertion analysis}

Initially, paired-end reads were filtered out by phred scores $<20$ and duplicate sequences were removed. After filtration, DNA fragments were consecutively mapped against the rice reference genome (phytozome v9 [14]) and T-vector sequence (Supplementary Fig. 1). The transgene insertion types were classified by adaptation and modification of the analytical strategies reported in Yang et al. [9]. Fig. 1 shows the workflow applied in this method. Initially, all NGS reads were individually mapped to the rice reference genome and transgenic vector (types A and C in Yang et al. [9]). Subsequently, these NGS reads were eliminated to conduct the following analyses. NGS reads not classified as above were classified into the following two classes: one side of the NGS read matched the reference genome, (1) the other one matched to vector (type B in Yang et al. [9]); or (2) one

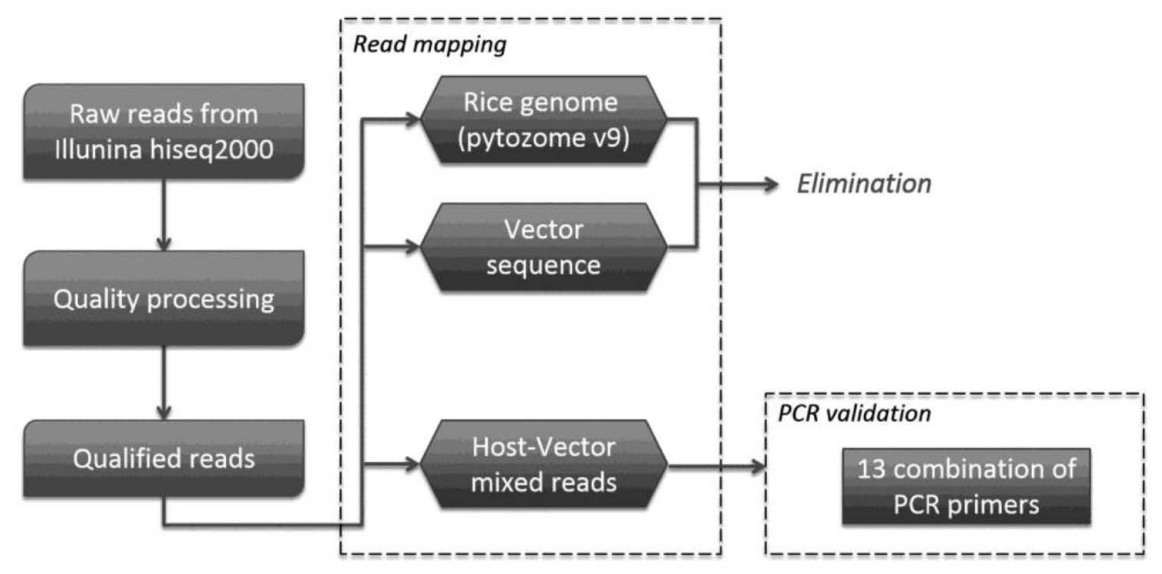

Fig. 1. Summary of the work-flow. $\mathrm{PCR}$, polymerase chain reaction. 
Table 1. Whole genome sequencing summary

\begin{tabular}{ccccc}
\hline Event & No. of reads & Total read length (bp) & Q30 (\%) & GC ratio (\%) \\
\hline PJKS131-2 & $194,965,440$ & $29,359,127,691(72 \times)$ & 71.56 & 41.58 \\
\hline
\end{tabular}

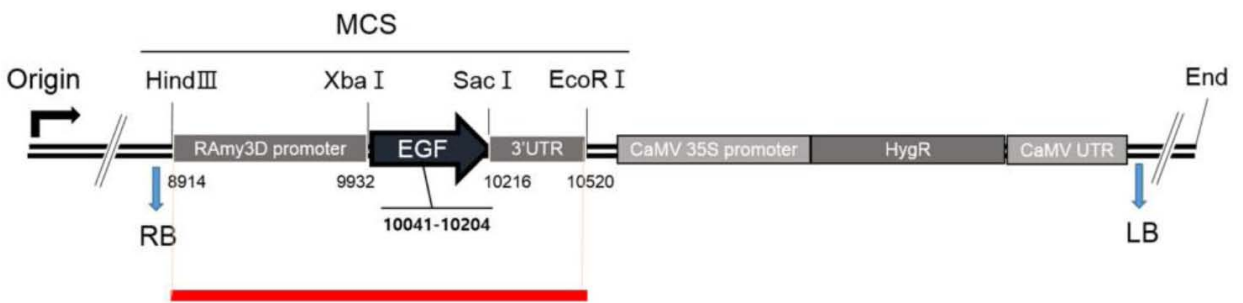

side of the NGS read exhibited both elements from the rice reference and transgenic vector (types D and E in Yang et al. [9]).

\section{Experimental validation of transgenic inserts}

Each of the 13 combination primer sets was designed congruent with the transgenic insertion region orientation. PCR was conducted using DNA polymerase (Solgent Co., Daejeon, Korea) following the manufacturer's instructions. The reaction was performed under the following conditions: a pre-denaturation step at $95^{\circ} \mathrm{C}$ for $5 \mathrm{~min}$; denaturation at $95^{\circ} \mathrm{C}$ for $60 \mathrm{~s} ; 30$ amplification cycles, including annealing at $60^{\circ} \mathrm{C}$ for $45 \mathrm{~s}$, and elongation at $72^{\circ} \mathrm{C}$ for $120 \mathrm{~s}$; and a final elongation at $72^{\circ} \mathrm{C}$ for $5 \mathrm{~min}$.

\section{Results}

\section{Whole genome re-sequencing and mapping to discover the transgenic position}

The transgenic GM rice site, PJKS131-2, was detected by performing whole genome re-sequencing using callus tissue. Genomic DNA libraries were constructed with an average $500 \mathrm{bp}$ and both ends were read with $150 \mathrm{bp}$ paired-end sequencing methods. A total length of raw sequencing reads were $29.3 \mathrm{~Gb}$ ( $\sim 194.9$ million reads), which showed $\sim 72 \times$ coverage in the total read length (Table 1$)$. Following quality control processing, reads with average phred scores $\geq 30$ were estimated at $\sim 71.5 \%$ (Table 1 ).

The types of mapped reads were classified by alignment of all NGS reads to the rice reference genome and transgenic vector sequences. Fig. 2 shows construction of the pJKS131 transgenic vector. Reads were aligned on the cloning vector positions $8,500 \mathrm{bp}$ to $10,500 \mathrm{bp}$, similar to transgenic insert locations. Detailed mapping strategies were described in the Methods. The transgene insertion site was identified by classifying reads where one end matched the host genome
Fig. 2. Schematic drawing of the transgenic vector genome. Red line represent the region of mapped reads. MCS, multiple cloning site; RB, right border; LB, left border.

\section{bp}

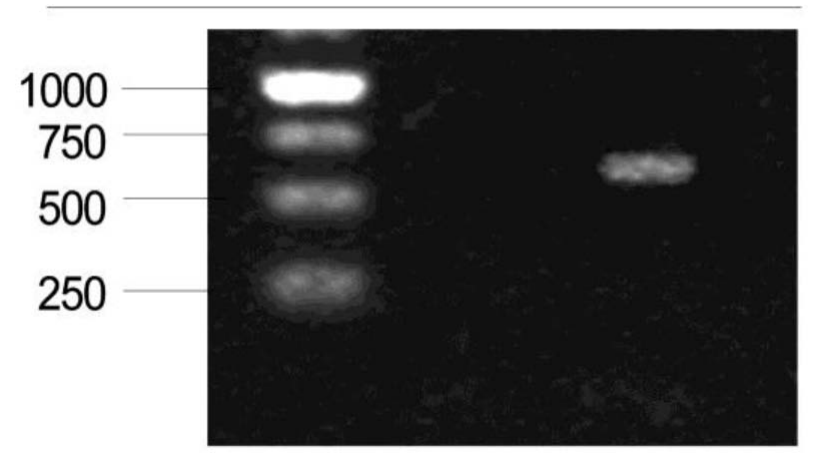

Fig. 3. Polymerase chain reaction validation of transgenic site.

and the other end matched the vector sequences (i.e., types $\mathrm{B}, \mathrm{D}$, and E) mapped back to the rice chromosome and known vector sequences. Eleven pairs of reads were identified on rice chromosome including chromosome 4. The total mapped reads described above were compatible with the transgenic vector backbone sequences.

\section{PCR validation of mapping prediction}

Thirteen PCR primers designed based on mapping direction validated the mapping results of 10 transgenic insert candidates. PCR results confirmed the target EGF sequence was successfully inserted on rice chromosome 4 (Figs. 3 and 4). The remaining reads were concluded to be artifacts, because all matches were not detected with PCR.

\section{Discussion}

Recent developments in NGS methods and accompanying bioinformatics tools have paved the way for ongoing genomics research widely used in the agricultural biotechnology field. Consequently, several studies reported new 


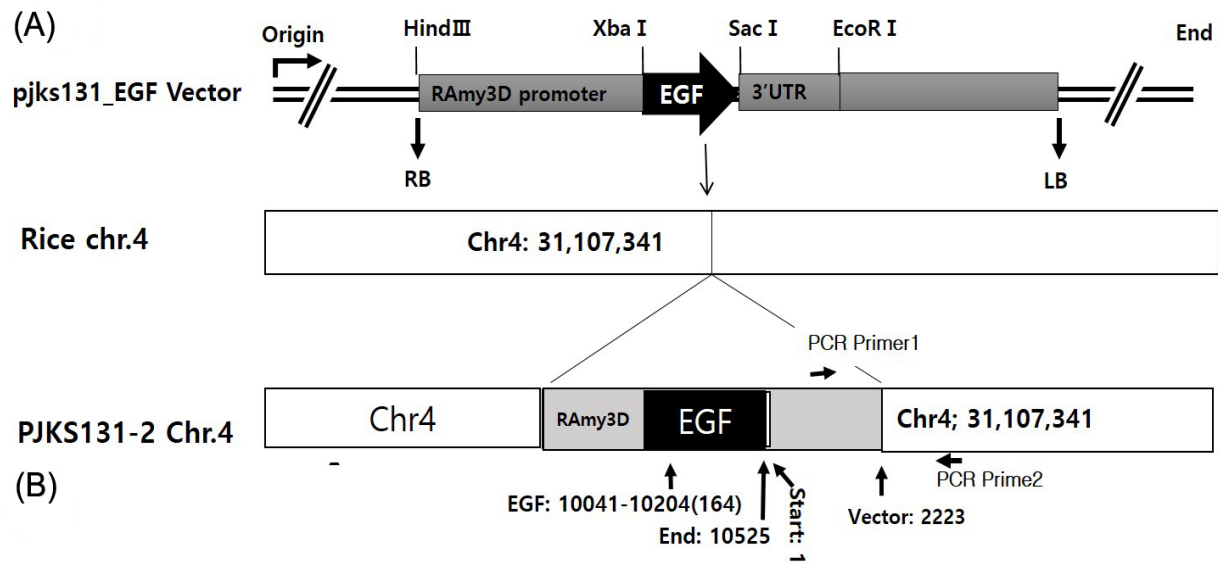

GAAGTACTCGCCGATAGTGGAAACCGACGCCCCAGCACTCGTCCGAGGGCAAAGAAATAGAGTAGATGCCGACCGGGATCTGTCG ATCGACAAGCTCGAGTTTCTCCATAATAATGTGTGAGTAGTTCCCAGATAAGGGAATTAGGGTTCCTATAGGGTTTCGCTCATGTGT TGAGCATATAAGAAACCCTTAGTATGTGATGCAAGTAAACAATTATGGATTCTACTTATGTTTCTTTTCCTTTTTCTGACTTGGGTTGCAGG TATGAGAAGAGGCATTCCAACATTCCGGCTCACGTCTCCCCATGCTTCCGTGTCAAGGAAGGTGACCATGTCATCATTGGCCAGTGCAGGT AAAACTTAACTCCCATACCTTAGTTTTTGACTCCTTAGAATCATCTTAAATAACAGAGGTGCTATTAGTTCAAACATTATCAGTTCACCCAGC TAGTACTATGAGATTTGGTTGCTGAAGTACAATTTCTGCATTTTCAACAGGCCGCTGTCGAAAACTGTGAGGTTCAACGTCCTGAAGGTCAT CCCAGCTGGATCCACCGGCGGCAGCGGCGGCAAGAAGGCCTTCACCG
Fig. 4. Transgenic position of epidermal growth factor $(E G F)$ locus on the rice chromosome 4 and polymerase chain reaction (PCR) test to identify T-DNA junction sequence. (A) The EGF is inserted on the position $31,104,341$ of the chromosome 4 . (B) The bold with underline is T-DNA sequence of the vector $2,026-2,223$ bp and the next bases is rice transgenic locus chromosome 4 (3110734131107690) in the fragment amplified by PCR test primer1 (5' TACCTGCATGCTGCGGTGAAG $3^{\prime}$ ) and primer2 (5`AGGGCTGTGTAGAAGTACTCGC $\left.3^{\prime}\right)$. approaches in GM crop safety assessment using NGS platforms [10-12]. In our study, we investigated EGF inserted GM rice events using NGS technology and bioinformatics to test the potential uses of this new approach in molecular assessment of transgenic organisms.

Results were successful in differentiating NGS read types using in silico analyses from GM rice, PJKS131-2 and hypothetically, the outcome was acceptable in terms of read classification. However, as a validation step, we experienced unexpected problems. Consistent with mapping and aligning data, we considered all possible transgenic insertion directions on the rice chromosomes and designed PCR primers based on loci information. Among the primers, except for locus specific primers on chromosome 4, results showed all matches were mismatches, which was caused by computational errors derived from analogous sequences between the rice genome and the transgenic vector. Therefore, we concluded it is essential to develop more accurate algorithms based on the transformation vector.

In addition, it is important to note our experimental sample was collected from rice callus tissues, with Agrobacterium co-incubation and a plant cell suspension culture system. Transgenic plant cell suspension culture system exhibits several advantages, including a low microorganism risk and chemical contamination, simple cell culture methods, economical facilities, and stable productivity. However, it is difficult to obtain pure genomic DNA of the host plant without plasmid DNA mixing using the plant cell culture method. We eliminated NGS raw reads mapped only against vector DNA (type $\mathrm{C}$ ), however if raw reads contained too many vector backbone sequences, problems in further bioinformatics analyses would still occur. Further studies are required with appropriate controls of GM plants in cell culture environments.

In the present study, we completed a proof-of-concept experiment to examine the molecular characterization of a recombinant-protein produced GM rice event using NGS methods. New approaches have recently been reported to assess the development and release of GM crops, however these techniques are not popularized in the field of GM risk assessment. However, previous studies in other disciplines have successfully established NGS, but for practical reasons, it has not been easy to apply this new method for testing GMOs. NGS strategies largely depend on sample quality, amount of data, and subsequent bioinformatics analyses. Therefore, it is critical proper guidelines to discovery transgenic site by NGS data matched and PCR test in the GMOs established and required.

\section{Supplementary material}

Supplementary data including one figure can be found with this article online at http://www.genominfo.org/src/ sm/gni-13-81-s001.pdf.

\section{Acknowledgments}

We thank members of bioinformatics team of National Instrumentation Center for Environmental management in Seoul National University. This work was supported by Next-Generation BioGreen21 program (PJ01131301), Rural Development Administration of the Korean government. 


\section{References}

1. Sabalza M, Christou P, Capell T. Recombinant plant-derived pharmaceutical proteins: current technical and economic bottlenecks. Biotechnol Lett 2014;36:2367-2379.

2. Schürch C, Blum P, Zülli F. Potential of plant cells in culture for cosmetic application. Phytochem Rev 2008;7:599-605.

3. Cohen SN, Chang AC, Boyer HW, Helling RB. Construction of biologically functional bacterial plasmids in vitro. Proc Natl Acad Sci U S A 1973;70:3240-3244.

4. European Parliament. Commission Implementing Regulation (EC) No 1829/2003 of the European Parliament and of the Council of 22 September 2003 on genetically modified food and feed. European Union Legislation. OJ L 268, 1-23. 2003.

5. European Parliament. REGULATION (EC) No 1830/2003 OF THE EUROPEAN PARLIAMENT AND OF THE COUNCIL of 22 September 2003 concerning the traceability and labelling of genetically modified organisms and the traceability of food and feed products produced from genetically modified organisms and amending Directive 2001/18/EC. European Union Legislation. OJ L 268, 24-28. 2003.

6. European Parliament. Commission Implementing Regulation (EU) No 503/2013 of 3 April 2013 on applications for authorisation of genetically modified food and feed in accordance with Regulation (EC) No 1829/2003 of the European Parliament and of the Council and amending Commission Regulations (EC) No 641/2004 and (EC) No 1981/2006. European Union Legislation. OJ L 157, 1-48. 2013.

7. Food and Agricultural Organization of the United Nations (FAO). Guideline for the conduct of food safety assessment of foods derived from recombinant-DNA plants. Rep. No.
CAC/GL 45-2003. Rome: FAO, 2003.

8. Food and Agricultural Organization of the United Nations (FAO). Guideline for the conduct of food safety assessment of foods derived from recombinant-DNA plants. Rep. No. CAC/GL. 68-2008. Rome: FAO, 2003.

9. Yang L, Wang C, Holst-Jensen A, Morisset D, Lin Y, Zhang D. Characterization of GM events by insert knowledge adapted re-sequencing approaches. Sci Rep 2013;3:2839.

10. Pauwels K, De Keersmaecker SC, De Schrijver A, du Jardin P, Roosens $\mathrm{NH}$, Herman P. Next-generation sequencing as a tool for the molecular characterisation and risk assessment of genetically modified plants: added value or not? Trends Food Sci Technol 2015;45:319-326.

11. Kovalic D, Garnaat C, Guo L, Yan Y, Groat J, Silvanovich A, et al. The use of next generation sequencing and junction sequence analysis bioinformatics to achieve molecular characterization of crops improved through modern biotechnology. Plant Genome 2012;5:149-163.

12. Wahler D, Schauser L, Bendiek J, Grohmann L. Next-generation sequencing as a tool for detailed molecular characterisation of genomic insertions and flanking regions in genetically modified plants: a pilot study using a rice event unauthorised in the EU. Food Anal Methods 2013;6:1718-1727.

13. Chan MT, Chang HH, Ho SL, Tong WF, Yu SM. Agrobacterium-mediated production of transgenic rice plants expressing a chimeric alpha-amylase promoter/beta-glucuronidase gene. Plant Mol Biol 1993;22:491-506.

14. Goodstein DM, Shu S, Howson R, Neupane R, Hayes RD, Fazo J, et al. Phytozome: a comparative platform for green plant genomics. Nucleic Acids Res 2012;40:D1178-D1186. 


\title{
SUPPLEMENTARY INFORMATION
}

\section{Efficiency to Discovery Transgenic Loci in GM Rice Using Next Generation Sequencing Whole Genome Re-sequencing}

\author{
Doori Park', Dongin Kim², Green Jang², Jongsung Lim², Yun-Ji Shin³, Jina Kim³,
} Mi-Seong Seo ${ }^{3}$, Su-Hyun Park, Ju-Kon Kim ${ }^{4}$, Tae-Ho Kwon ${ }^{3 *}$, Ik-Young Choi ${ }^{1 * *}$

${ }^{1}$ Institute of Green Bio Science and Technology, Seoul National University, Pyeongchang 25354, Korea,

${ }^{2}$ National Instrumentation Center for Environmental Management, College of Agriculture and Life Sciences,

Seoul National University, Seoul 08826, Korea,

${ }^{3}$ Natural Bio-Materials Inc., Wanju 55322, Korea,

${ }^{4}$ Crop Biotech Institute, Green-Bio Science and Technology, Seoul National University, Pyeongchang 25354, Korea 


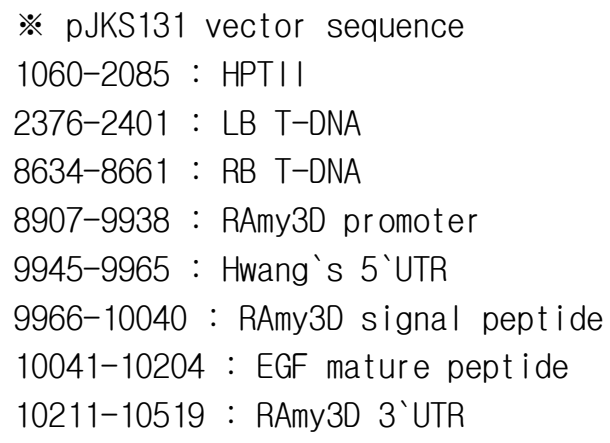

$\begin{array}{llllll}10 & 20 & 30 & 40 & 50 & 60\end{array}$

GTAATCATGG TCATAGCTGT TTCCTGTGTG AAATTGTTAT CCGCTCACAA TTCCACACAA

$\begin{array}{llllll}70 & 80 & 90 & 100 & 110 & 120\end{array}$
CATACGAGCC GGAAGCATAA AGTGTAAAGC CTGGGGTGCC TAATGAGTGA GCTAACTCAC

$\begin{array}{llllll}130 & 140 & 150 & 160 & 170 & 180\end{array}$ ATTAATTGCG TTGCGCTCAC TGCCCGCTTT CCAGTCGGGA AACCTGTCGT GCCAGCTGCA $\begin{array}{lllll}190 & 200 & 210 & 220 & 230\end{array}$ TTAATGAATC GGCCAACGCG CGGGGAGAGG CGGTTTGCGT ATTGGCTAGA GCAGCTTGCC

$\begin{array}{llllll}250 & 260 & 270 & 280 & 290 & 300\end{array}$
AACATGGTGG AGCACGACAC TCTCGTCTAC TCCAAGAATA TCAAAGATAC AGTCTCAGAA

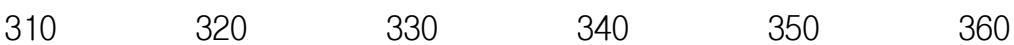
GACCAAAGGG CTATTGAGAC TTTTCAACAA AGGGTAATAT CGGGAAACCT CCTCGGATTC

$\begin{array}{llllll}370 & 380 & 390 & 400 & 410 & 420\end{array}$
CATTGCCCAG CTATCTGTCA CTTCATCAAA AGGACAGTAG AAAAGGAAGG TGGCACCTAC

$\begin{array}{llllll}430 & 440 & 450 & 460 & 470 & 480\end{array}$
AAATGCCATC ATTGCGATAA AGGAAAGGCT ATCGTTCAAG ATGCCTCTGC CGACAGTGGT

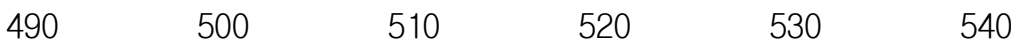
CCCAAAGATG GACCCCCACC CACGAGGAGC ATCGTGGAAA AAGAAGACGT TCCAACCACG

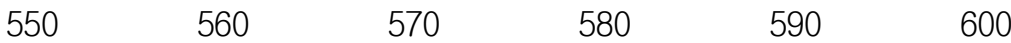
TCTTCAAAGC AAGTGGATTG ATGTGATAAC ATGGTGGAGC ACGACACTCT CGTCTACTCC

$\begin{array}{llllll}610 & 620 & 630 & 640 & 650 & 660\end{array}$ AAGAATATCA AAGATACAGT CTCAGAAGAC CAAAGGGCTA TTGAGACTTT TCAACAAAGG

$\begin{array}{llllll}670 & 680 & 690 & 700 & 710 & 720\end{array}$
GTAATATCGG GAAACCTCCT CGGATTCCAT TGCCCAGCTA TCTGTCACTT CATCAAAAGG

$\begin{array}{llllll}730 & 740 & 750 & 760 & 770 & 780\end{array}$ ACAGTAGAAA AGGAAGGTGG CACCTACAAA TGCCATCATT GCGATAAAGG AAAGGCTATC 
$\begin{array}{llllll}790 & 800 & 810 & 820 & 830 & 840\end{array}$ GTTCAAGATG CCTCTGCCGA CAGTGGTCCC AAAGATGGAC CCCCACCCAC GAGGAGCATC $\begin{array}{llllll}850 & 860 & 870 & 880 & 890 & 900\end{array}$ GTGGAAAAAG AAGACGTTCC AACCACGTCT TCAAAGCAAG TGGATTGATG TGATATCTCC

$\begin{array}{llllll}910 & 920 & 930 & 940 & 950 & 960\end{array}$
ACTGACGTAA GGGATGACGC ACAATCCCAC TATCCTTCGC AAGACCTTCC TCTATATAAG $\begin{array}{llllll}970 & 980 & 990 & 1000 & 1010 & 1020\end{array}$ GAAGTTCATT TCATTTGGAG AGGACACGCT GAAATCACCA GTCTCTCTCT ACAAATCTAT $\begin{array}{llllll}1030 & 1040 & 1050 & 1060 & 1070 & 1080\end{array}$ CTCTCTCGAG CTTTCGCAGA TCCCGGGGGG CAATGAGATA TGAAAAAGCC TGAACTCACC

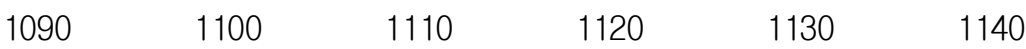
GCGACGTCTG TCGAGAAGTT TCTGATCGAA AAGTTCGACA GCGTCTCCGA CCTGATGCAG $\begin{array}{llllll}1150 & 1160 & 1170 & 1180 & 1190 & 1200\end{array}$ CTCTCGGAGG GCGAAGAATC TCGTGCTTTC AGCTTCGATG TAGGAGGGCG TGGATATGTC $\begin{array}{llllll}1210 & 1220 & 1230 & 1240 & 1250 & 1260\end{array}$ CTGCGGGTAA ATAGCTGCGC CGATGGTTTC TACAAAGATC GTTATGTTTA TCGGCACTTT $\begin{array}{llllll}1270 & 1280 & 1290 & 1300 & 1310 & 1320\end{array}$ GCATCGGCCG CGCTCCCGAT TCCGGAAGTG CTTGACATTG GGGAGTTTAG CGAGAGCCTG

$\begin{array}{llllll}1330 & 1340 & 1350 & 1360 & 1370 & 1380\end{array}$
ACCTATTGCA TCTCCCGCCG TGCACAGGGT GTCACGTTGC AAGACCTGCC TGAAACCGAA

$\begin{array}{llllll}1390 & 1400 & 1410 & 1420 & 1430 & 1440\end{array}$
CTGCCCGCTG TTCTACAACC GGTCGCGGAG GCTATGGATG CGATCGCTGC GGCCGATCTT $\begin{array}{llllll}1450 & 1460 & 1470 & 1480 & 1490 & 1500\end{array}$ AGCCAGACGA GCGGGTTCGG CCCATTCGGA CCGCAAGGAA TCGGTCAATA CACTACATGG

$\begin{array}{llllll}1510 & 1520 & 1530 & 1540 & 1550 & 1560\end{array}$
CGTGATTTCA TATGCGCGAT TGCTGATCCC CATGTGTATC ACTGGCAAAC TGTGATGGAC $\begin{array}{llllll}1570 & 1580 & 1590 & 1600 & 1610 & 1620\end{array}$ GACACCGTCA GTGCGTCCGT CGCGCAGGCT CTCGATGAGC TGATGCTTTG GGCCGAGGAC $\begin{array}{llllll}1630 & 1640 & 1650 & 1660 & 1670 & 1680\end{array}$ TGCCCCGAAG TCCGGCACCT CGTGCACGCG GATTTCGGCT CCAACAATGT CCTGACGGAC $\begin{array}{llllll}1690 & 1700 & 1710 & 1720 & 1730 & 1740\end{array}$ AATGGCCGCA TAACAGCGGT CATTGACTGG AGCGAGGCGA TGTTCGGGGA TTCCCAATAC 
$\begin{array}{llllll}1750 & 1760 & 1770 & 1780 & 1790 & 1800\end{array}$ GAGGTCGCCA ACATCTTCTT CTGGAGGCCG TGGTTGGCTT GTATGGAGCA GCAGACGCGC $\begin{array}{llllll}1810 & 1820 & 1830 & 1840 & 1850 & 1860\end{array}$ TACTTCGAGC GGAGGCATCC GGAGCTTGCA GGATCGCCAC GACTCCGGGC GTATATGCTC

$\begin{array}{llllll}1870 & 1880 & 1890 & 1900 & 1910 & 1920\end{array}$
CGCATTGGTC TTGACCAACT CTATCAGAGC TTGGTTGACG GCAATTTCGA TGATGCAGCT $\begin{array}{llllll}1930 & 1940 & 1950 & 1960 & 1970 & 1980\end{array}$ TGGGCGCAGG GTCGATGCGA CGCAATCGTC CGATCCGGAG CCGGGACTGT CGGGCGTACA

$\begin{array}{lllll}1990 & 2000 & 2010 & 2020 & 2030\end{array} 2040$
CAAATCGCCC GCAGAAGCGC GGCCGTCTGG ACCGATGGCT GTGTAGAAGT ACTCGCCGAT

$2050 \quad 2060 \quad 2070 \quad 2080 \quad 2090 \quad 2100$
AGTGGAAACC GACGCCCCAG CACTCGTCCG AGGGCAAAGA AATAGAGTAG ATGCCGACCG

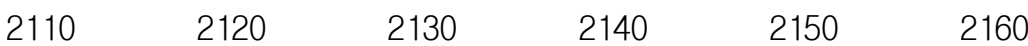
GATCTGTCGA TCGACAAGCT CGAGTTTCTC CATAATAATG TGTGAGTAGT TCCCAGATAA

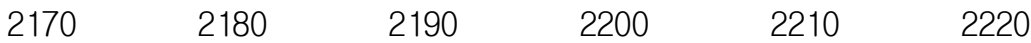
GGGAATTAGG GTTCCTATAG GGTTTCGCTC ATGTGTTGAG CATATAAGAA ACCCTTAGTA

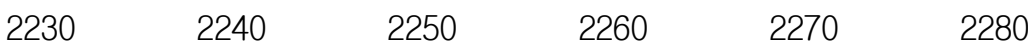
TGTATTTGTA TTTGTAAAAT ACTTCTATCA ATAAAATTTC TAATTCCTAA AACCAAAATC $2290 \quad 2300 \quad 2310 \quad 2320 \quad 2330 \quad 2340$ CAGTACTAAA ATCCAGATCC CCCGAATTAA TTCGGCGTTA ATTCAGTACA TTAAAAACGT

$\begin{array}{llllll}2350 & 2360 & 2370 & 2380 & 2390 & 2400\end{array}$
CCGCAATGTG TTATTAAGTT GTCTAAGCGT CAATTTGTTT ACACCACAAT ATATCCTGCC

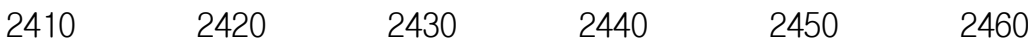
ACCAGCCAGC CAACAGCTCC CCGACCGGCA GCTCGGCACA AAATCACCAC TCGATACAGG $2470 \quad 2480 \quad 2490 \quad 2500 \quad 2510 \quad 2520$ CAGCCCATCA GTCCGGGACG GCGTCAGCGG GAGAGCCGTT GTAAGGCGGC AGACTTTGCT $\begin{array}{llllll}2530 & 2540 & 2550 & 2560 & 2570 & 2580\end{array}$ CATGTTACCG ATGCTATTCG GAAGAACGGC AACTAAGCTG CCGGGTTTGA AACACGGATG $2590 \quad 2600 \quad 2610 \quad 2620 \quad 2630 \quad 2640$ ATCTCGCGGA GGGTAGCATG TTGATTGTAA CGATGACAGA GCGTTGCTGC CTGTGATCAC

$2650 \quad 2660 \quad 2670 \quad 2680 \quad 2690 \quad 2700$
CGCGGTTTCA AAATCGGCTC CGTCGATACT ATGTTATACG CCAACTTTGA AAACAACTTT 
$\begin{array}{llrrr}2710 & 2720 & 2730 & 2740 & 2750\end{array}$

GAAAAGCTG TTTTCTGGTA TTTAAgGTTT TAGAATGCAA GGAACAGTGA ATTGGAGTTC

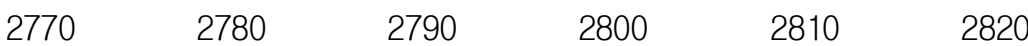

GTCTTGTTAT AATTAGCTTC TTGGGGTATC TTTAAATACT GTAGAAAAGA GGAAGGAAAT

$2830 \quad 2840 \quad 2850 \quad 2860 \quad 2870 \quad 2880$

AATAAATGGC TAAAATGAGA ATATCACCGG AATTGAAAAA ACTGATCGAA AAATACCGCT

$\begin{array}{lllrr}2890 & 2900 & 2910 & 2920 & 2930\end{array}$

GCGTAAAAGA TACGGAAGGA ATGTCTCCTG CTAAGGTATA TAAGCTGGTG GGAGAAAATG

$2950 \quad 2960 \quad 2970 \quad 2980 \quad 3000$

AAAACCTATA TTTAAAAATG ACGGACAGCC GGTATAAAGG GACCACCTAT GATGTGGAAC

$\begin{array}{lllll}3010 & 3020 & 3030 & 3040 & 3050\end{array} 3060$

GGGAAAGGA CATGATGCTA TGGCTGGAAG GAAAGCTGCC TGTTCCAAAg GTCCTGCACT

$\begin{array}{lllll}3070 & 3080 & 3090 & 3100 & 3110\end{array}$

TTGAACGGCA TGATGGCTGG AGCAATCTGC TCATGAGTGA GGCCGATGGC GTCCTTTGCT

$\begin{array}{lllll}3130 & 3140 & 3150 & 3160 & 3170\end{array}$

CGGAAGAGTA TGAAGATGAA CAAAGCCCTG AAAAGATTAT CGAGCTGTAT GCGGAGTGCA

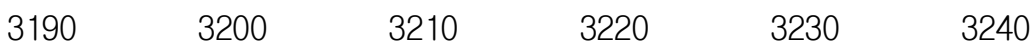

TCAGGCTCTT TCACTCCATC GACATATCGG ATTGTCCCTA TACGAATAGC TTAGACAGCC

$\begin{array}{llllr}3250 & 3260 & 3270 & 3280 & 3290\end{array}$

GCTTAGCCGA ATTGGATTAC TTACTGAATA ACGATCTGGC CGATGTGGAT TGCGAAAACT

$\begin{array}{lllll}3310 & 3320 & 3330 & 3340 & 3350\end{array}$

GGGAAGAAGA CACTCCATTT AAAGATCCGC GCGAGCTGTA TGATTTTTTA AAGACGGAAA

$\begin{array}{lllll}3370 & 3380 & 3390 & 3400 & 3410\end{array}$

AGCCCGAAGA GGAACTTGTC TTTTCCCACG GCGACCTGGG AGACAGCAAC ATCTTTGTGA

$\begin{array}{lllll}3430 & 3440 & 3450 & 3460 & 3470\end{array}$

AAGATGGCAA AGTAAGTGGC TTTATTGATC TTGGGAGAAG CGGCAGGGCG GACAAGTGGT

$\begin{array}{lllll}3490 & 3500 & 3510 & 3520 & 3530\end{array}$

ATGACATTGC CTTCTGCGTC CGGTCGATCA GGGAGGATAT CGGGGAAGA CAGTATGTCG

$3550 \quad 3560 \quad 3570 \quad 3580 \quad 3590 \quad 3600$

AGCTATTTTT TGACTTACTG GGGATCAAGC CTGATTGGGA GAAAATAAAA TATTATATTT

$\begin{array}{lllll}3610 & 3620 & 3630 & 3640 & 3650\end{array}$

TACTGGATGA ATTGTTTTAG TACCTAGAAT GCATGACCAA AATCCCTTAA CGTGAGTTTT 
$\begin{array}{lllll}3670 & 3680 & 3690 & 3700 & 3710\end{array}$

CGTTCCACTG AGCGTCAGAC CCCGTAGAAA AGATCAAAGG ATCTTCTTGA GATCCTTTTT

$\begin{array}{lllll}3730 & 3740 & 3750 & 3760 & 3770\end{array}$

TTCTGCGCGT AATCTGCTGC TTGCAAACAA AAAAACCACC GCTACCAGCG GTGGTTTGTT

$\begin{array}{lllll}3790 & 3800 & 3810 & 3820 & 3830\end{array}$

TGCCGGATCA AGAGCTACCA ACTCTTTTTC CGAAGGTAAC TGGCTTCAGC AGAGCGCAGA

$\begin{array}{lllll}3850 & 3860 & 3870 & 3880 & 3890\end{array}$

TACCAAATAC TGTCCTTCTA GTGTAGCCGT AGTTAGGCCA CCACTTCAAG AACTCTGTAG

$\begin{array}{lllll}3910 & 3920 & 3930 & 3940 & 3950\end{array}$

CACCGCCTAC ATACCTCGCT CTGCTAATCC TGTTACCAGT GGCTGCTGCC AGTGGCGATA

$\begin{array}{lllll}3970 & 3980 & 3990 & 4000 & 4010\end{array}$

AGTCGTGTCT TACCGGGTTG GACTCAAGAC GATAGTTACC GGATAAGGCG CAGCGGTCGG

$\begin{array}{lllll}4030 & 4040 & 4050 & 4060 & 4070\end{array}$

GCTGAACGGG GGGTTCGTGC ACACAGCCCA GCTTGGAGCG AACGACCTAC ACCGAACTGA

$4090 \quad 4100 \quad 4110 \quad 4120 \quad 4130 \quad 4140$

GATACCTACA GCGTGAGCTA TGAGAAAGCG CCACGCTTCC CGAAGGGAGA AAGGCGGACA

$\begin{array}{lllll}4150 & 4160 & 4170 & 4180 & 4190\end{array}$

GGTATCCGGT AAGCGGCAGG GTCGGAACAG GAGAGCGCAC GAGGGAGCTT CCAGGGGGAA

$\begin{array}{lllll}4210 & 4220 & 4230 & 4240 & 4250\end{array}$

ACGCCTGGTA TCTTTATAGT CCTGTCGGGT TTCGCCACCT CTGACTTGAG CGTCGATTTT

$\begin{array}{lllll}4270 & 4280 & 4290 & 4300 & 4310\end{array}$

TGTGATGCTC GTCAGGGGGG CGGAGCCTAT GGAAAAACGC CAGCAACGCG GCCTTTTTAC

$\begin{array}{lllll}4330 & 4340 & 4350 & 4360 & 4370\end{array}$

GGTTCCTGGC CTTTTGCTGG CCTTTTGCTC ACATGTTCTT TCCTGCGTTA TCCCCTGATT

$\begin{array}{lllll}4390 & 4400 & 4410 & 4420 & 4430\end{array}$

CTGTGGATAA CCGTATTACC GCCTTTGAGT GAGCTGATAC CGCTCGCCGC AGCCGAACGA

$\begin{array}{lllll}4450 & 4460 & 4470 & 4480 & 4490\end{array}$

CCGAGCGCAG CGAGTCAGTG AGCGAGGAAG CGGAAGAGCG CCTGATGCGG TATTTTCTCC

$\begin{array}{lllll}4510 & 4520 & 4530 & 4540 & 4550\end{array}$

TTACGCATCT GTGCGGTATT TCACACCGCA TATGGTGCAC TCTCAGTACA ATCTGCTCTG

$\begin{array}{lllll}4570 & 4580 & 4590 & 4600 & 4610\end{array}$

ATGCCGCATA GTTAAGCCAG TATACACTCC GCTATCGCTA CGTGACTGGG TCATGGCTGC 
$\begin{array}{lllll}4630 & 4640 & 4650 & 4660 & 4670\end{array} 4680$ GCCCCGACAC CCGCCAACAC CCGCTGACGC GCCCTGACGG GCTTGTCTGC TCCCGGCATC $\begin{array}{llllll}4690 & 4700 & 4710 & 4720 & 4730 & 4740\end{array}$ CGCTTACAGA CAAGCTGTGA CCGTCTCCGG GAGCTGCATG TGTCAGAGGT TTTCACCGTC $\begin{array}{llllll}4750 & 4760 & 4770 & 4780 & 4790 & 4800\end{array}$ ATCACCGAAA CGCGCGAGGC AGGGTGCCTT GATGTGGGCG CCGGCGGTCG AGTGGCGACG $4810 \quad 4820 \quad 4830 \quad 4840 \quad 4850 \quad 4860$ GCGCGGCTTG TCCGCGCCCT GGTAGATTGC CTGGCCGTAG GCCAGCCATT TTTGAGCGGC $4870 \quad 4880 \quad 4890 \quad 4900 \quad 4910 \quad 4920$ CAGCGGCCGC GATAGGCCGA CGCGAAGCGG CGGGGCGTAG GGAGCGCAGC GACCGAAGGG

$\begin{array}{llllll}4930 & 4940 & 4950 & 4960 & 4970 & 4980\end{array}$ TAGGCGCTTT TTGCAGCTCT TCGGCTGTGC GCTGGCCAGA CAGTTATGCA CAGGCCAGGC

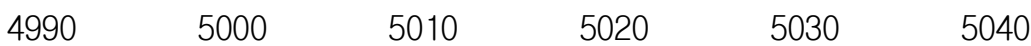
GGGTTTTAAG AGTTTTAATA AGTTTTAAAG AGTTTTAGGC GGAAAAATCG CCTTTTTTCT

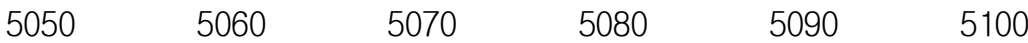
CTTTTATATC AGTCACTTAC ATGTGTGACC GGTTCCCAAT GTACGGCTTT GGGTTCCCAA

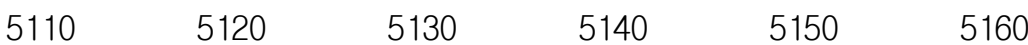
TGTACGGGTT CCGGTTCCCA ATGTACGGCT TTGGGTTCCC AATGTACGTG CTATCCACAG

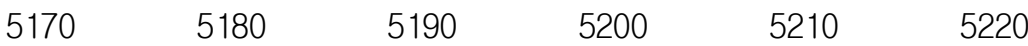
GAAAGAGACC TTTTCGACCT TTTTCCCCTG CTAGGGCAAT TTGCCCTAGC ATCTGCTCCG

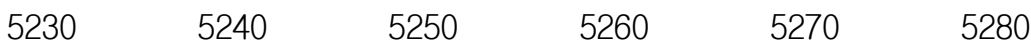
TACATTAGGA ACCGGCGGAT GCTTCGCCCT CGATCAGGTT GCGGTAGCGC ATGACTAGGA $5290 \quad 5300 \quad 5310 \quad 5320 \quad 5330 \quad 5340$ TCGGGCCAGC CTGCCCCGCC TCCTCCTTCA AATCGTACTC CGGCAGGTCA TTTGACCCGA

$5350 \quad 5360 \quad 5370 \quad 5380 \quad 5390 \quad 5400$
TCAGCTTGCG CACGGTGAAA CAGAACTTCT TGAACTCTCC GGCGCTGCCA CTGCGTTCGT $5410 \quad 5420 \quad 5430 \quad 5440 \quad 5450 \quad 5460$ AGATCGTCTT GAACAACCAT CTGGCTTCTG CCTTGCCTGC GGCGCGGCGT GCCAGGCGGT $5470 \quad 5480 \quad 5490 \quad 5500 \quad 5510 \quad 5520$ AGAGAAAACG GCCGATGCCG GGATCGATCA AAAAGTAATC GGGGTGAACC GTCAGCACGT

$5530 \quad 5540 \quad 5550 \quad 5560 \quad 5570 \quad 5580$ CCGGGTTCTT GCCTTCTGTG ATCTCGCGGT ACATCCAATC AGCTAGCTCG ATCTCGATGT 


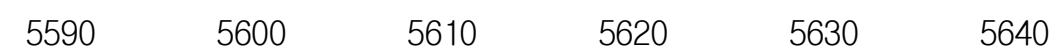

ACTCCGGCCG CCCGGTTTCG CTCTTTACGA TCTTGTAGCG GCTAATCAAG GCTTCACCCT

$5650 \quad 5660 \quad 5670 \quad 5680 \quad 5690 \quad 5700$

CGGATACCGT CACCAGGCGG CCGTTCTTGG CCTTCTTCGT ACGCTGCATG GCAACGTGCG

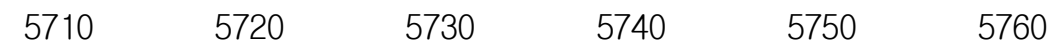

TGGTGTTTAA CCGAATGCAG GTTTCTACCA GGTCGTCTTT CTGCTTTCCG CCATCGGCTC

$5770 \quad 5780 \quad 5790 \quad 5800 \quad 5810 \quad 5820$

GCCGGCAGAA CTTGAGTACG TCCGCAACGT GTGGACGGAA CACGCGGCCG GGCTTGTCTC

$5830 \quad 5840 \quad 5850 \quad 5860 \quad 5870 \quad 5880$

CCTTCCCTTC CCGGTATCGG TTCATGGATT CGGTTAGATG GGAAACCGCC ATCAGTACCA

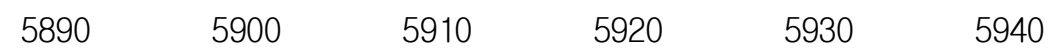

GGTCGTAATC CCACACACTG GCCATGCCGG CCGGCCCTGC GGAAACCTCT ACGTGCCCGT

$5950 \quad 5960 \quad 5970 \quad 5980 \quad 5990 \quad 6000$

CTGGAAGCTC GTAGCGGATC ACCTCGCCAG CTCGTCGGTC ACGCTTCGAC AGACGGAAAA

$\begin{array}{llllll}6010 & 6020 & 6030 & 6040 & 6050 & 6060\end{array}$

CGGCCACGTC CATGATGCTG CGACTATCGC GGGTGCCCAC GTCATAGAGC ATCGGAACGA

$\begin{array}{llllll}6070 & 6080 & 6090 & 6100 & 6110 & 6120\end{array}$

AAAAATCTGG TTGCTCGTCG CCCTTGGGCG GCTTCCTAAT CGACGGCGCA CCGGCTGCCG

$\begin{array}{llllll}6130 & 6140 & 6150 & 6160 & 6170 & 6180\end{array}$

GCGGTTGCCG GGATTCTTTG CGGATTCGAT CAGCGGCCGC TTGCCACGAT TCACCGGGGC

$\begin{array}{lllll}6190 & 6200 & 6210 & 6220 & 6230\end{array} 6240$

GTGCTTCTGC CTCGATGCGT TGCCGCTGGG CGGCCTGCGC GGCCTTCAAC TTCTCCACCA

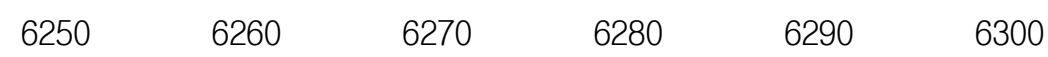

GGTCATCACC CAGCGCCGCG CCGATTTGTA CCGGGCCGGA TGGTTTGCGA CCGTCACGCC

$\begin{array}{lllll}6310 & 6320 & 6330 & 6340 & 6350\end{array} 6360$

GATTCCTCGG GCTTGGGGGT TCCAGTGCCA TTGCAGGGCC GGCAGACAAC CCAGCCGCTT

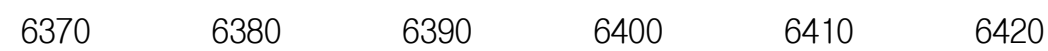

ACGCCTGGCC AACCGCCCGT TCCTCCACAC ATGGGGCATT CCACGGCGTC GGTGCCTGGT

$\begin{array}{lllll}6430 & 6440 & 6450 & 6460 & 6470\end{array} 6480$

TGTTCTTGAT TTTCCATGCC GCCTCCTTTA GCCGCTAAAA TTCATCTACT CATTTATTCA

$\begin{array}{llllll}6490 & 6500 & 6510 & 6520 & 6530 & 6540\end{array}$

TTTGCTCATT TACTCTGGTA GCTGCGCGAT GTATTCAGAT AGCAGCTCGG TAATGGTCTT 
$\begin{array}{llllll}6550 & 6560 & 6570 & 6580 & 6590 & 6600\end{array}$ GCCTTGGCGT ACCGCGTACA TCTTCAGCTT GGTGTGATCC TCCGCCGGCA ACTGAAAGTT

$\begin{array}{llllll}6610 & 6620 & 6630 & 6640 & 6650 & 6660\end{array}$ GACCCGCTTC ATGGCTGGCG TGTCTGCCAG GCTGGCCAAC GTTGCAGCCT TGCTGCTGCG

$\begin{array}{llllll}6670 & 6680 & 6690 & 6700 & 6710 & 6720\end{array}$
TGCGCTCGGA CGGCCGGCAC TTAGCGTGTT TGTGCTTTTG CTCATTTTCT CTTTACCTCA
6730
6740
6750
6760
6770
6780

TTAACTCAAA TGAGTTTTGA TTTAATTTCA GCGGCCAGCG CCTGGACCTC GCGGGCAGCG

$\begin{array}{llllll}6790 & 6800 & 6810 & 6820 & 6830 & 6840\end{array}$ TCGCCCTCGG GTTCTGATTC AAGAACGGTT GTGCCGGCGG CGGCAGTGCC TGGGTAGCTC

$\begin{array}{llllll}6850 & 6860 & 6870 & 6880 & 6890 & 6900\end{array}$
ACGCGCTGCG TGATACGGGA CTCAAGAATG GGCAGCTCGT ACCCGGCCAG CGCCTCGGCA

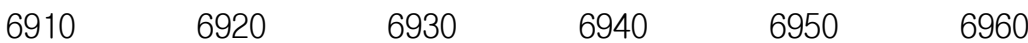
ACCTCACCGC CGATGCGCGT GCCTTTGATC GCCCGCGACA CGACAAAGGC CGCTTGTAGC

$\begin{array}{llllll}6970 & 6980 & 6990 & 7000 & 7010 & 7020\end{array}$
CTTCCATCCG TGACCTCAAT GCGCTGCTTA ACCAGCTCCA CCAGGTCGGC GGTGGCCCAT

$\begin{array}{llllll}7030 & 7040 & 7050 & 7060 & 7070 & 7080\end{array}$
ATGTCGTAAG GGCTTGGCTG CACCGGAATC AGCACGAAGT CGGCTGCCTT GATCGCGGAC

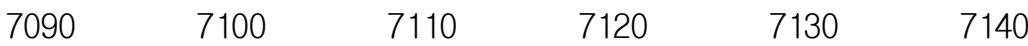
ACAGCCAAGT CCGCCGCCTG GGGCGCTCCG TCGATCACTA CGAAGTCGCG CCGGCCGATG

$\begin{array}{llllll}7150 & 7160 & 7170 & 7180 & 7190 & 7200\end{array}$
GCCTTCACGT CGCGGTCAAT CGTCGGGCGG TCGATGCCGA CAACGGTTAG CGGTTGATCT

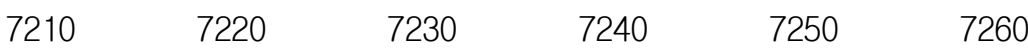
TCCCGCACGG CCGCCCAATC GCGGGCACTG CCCTGGGGAT CGGAATCGAC TAACAGAACA

$\begin{array}{llllll}7270 & 7280 & 7290 & 7300 & 7310 & 7320\end{array}$
TCGGCCCCGG CGAGTTGCAG GGCGCGGGCT AGATGGGTTG CGATGGTCGT CTTGCCTGAC $\begin{array}{llllll}7330 & 7340 & 7350 & 7360 & 7370 & 7380\end{array}$ CCGCCTTTCT GGTTAAGTAC AGCGATAACC TTCATGCGTT CCCCTTGCGT ATTTGTTTAT $\begin{array}{llllll}7390 & 7400 & 7410 & 7420 & 7430 & 7440\end{array}$ TTACTCATCG CATCATATAC GCAGCGACCG CATGACGCAA GCTGTTTTAC TCAAATACAC

$\begin{array}{llllll}7450 & 7460 & 7470 & 7480 & 7490 & 7500\end{array}$ ATCACCTTTT TAGACGGCGG CGCTCGGTTT CTTCAGCGGC CAAGCTGGCC GGCCAGGCCG 


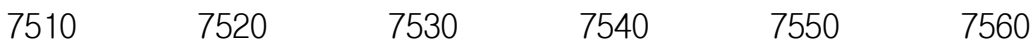

CCAGCTTGGC ATCAGACAAA CCGGCCAGGA TTTCATGCAG CCGCACGGTT GAGACGTGCG

$\begin{array}{llllll}7570 & 7580 & 7590 & 7600 & 7610 & 7620\end{array}$

CGGGCGGCTC GAACACGTAC CCGGCCGCGA TCATCTCCGC CTCGATCTCT TCGGTAATGA

$\begin{array}{llllll}7630 & 7640 & 7650 & 7660 & 7670 & 7680\end{array}$

AAAACGGTTC GTCCTGGCCG TCCTGGTGCG GTTTCATGCT TGTTCCTCTT GGCGTTCATT

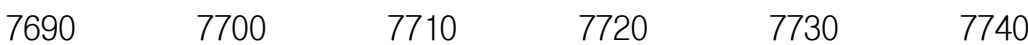

CTCGGCGGCC GCCAGGGCGT CGGCCTCGGT CAATGCGTCC TCACGGAAGG CACCGCGCCG

$\begin{array}{llllll}7750 & 7760 & 7770 & 7780 & 7790 & 7800\end{array}$

CCTGGCCTCG GTGGGCGTCA CTTCCTCGCT GCGCTCAAGT GCGCGGTACA GGGTCGAGCG

$\begin{array}{llllll}7810 & 7820 & 7830 & 7840 & 7850 & 7860\end{array}$

ATGCACGCCA AGCAGTGCAG CCGCCTCTTT CACGGTGCGG CCTTCCTGGT CGATCAGCTC

$\begin{array}{llllll}7870 & 7880 & 7890 & 7900 & 7910 & 7920\end{array}$

GCGGGCGTGC GCGATCTGTG CCGGGGTGAG GGTAGGGCGG GGGCCAAACT TCACGCCTCG

$\begin{array}{llllll}7930 & 7940 & 7950 & 7960 & 7970 & 7980\end{array}$

GGCCTTGGCG GCCTCGCGCC CGCTCCGGGT GCGGTCGATG ATTAGGGAAC GCTCGAACTC

$\begin{array}{llllll}7990 & 8000 & 8010 & 8020 & 8030 & 8040\end{array}$

GGCAATGCCG GCGAACACGG TCAACACCAT GCGGCCGGCC GGCGTGGTGG TGTCGGCCCA

$\begin{array}{llllll}8050 & 8060 & 8070 & 8080 & 8090 & 8100\end{array}$

CGGCTCTGCC AGGCTACGCA GGCCCGCGCC GGCCTCCTGG ATGCGCTCGG CAATGTCCAG

$\begin{array}{llllll}8110 & 8120 & 8130 & 8140 & 8150 & 8160\end{array}$

TAGGTCGCGG GTGCTGCGGG CCAGGCGGTC TAGCCTGGTC ACTGTCACAA CGTCGCCAGG

$\begin{array}{llllll}8170 & 8180 & 8190 & 8200 & 8210 & 8220\end{array}$

GCGTAGGTGG TCAAGCATCC TGGCCAGCTC CGGGCGGTCG CGCCTGGTGC CGGTGATCTT

$\begin{array}{llllll}8230 & 8240 & 8250 & 8260 & 8270 & 8280\end{array}$

CTCGGAAAAC AGCTTGGTGC AGCCGGCCGC GTGCAGTTCG GCCCGTTGGT TGGTCAAGTC

$\begin{array}{llllll}8290 & 8300 & 8310 & 8320 & 8330 & 8340\end{array}$

CTGGTCGTCG GTGCTGACGC GGGCATAGCC CAGCAGGCCA GCGGCGGCGC TCTTGTTCAT

$\begin{array}{llllll}8350 & 8360 & 8370 & 8380 & 8390 & 8400\end{array}$

GGCGTAATGT CTCCGGTTCT AGTCGCAAGT ATTCTACTTT ATGCGACTAA AACACGCGAC

$\begin{array}{llllll}8410 & 8420 & 8430 & 8440 & 8450 & 8460\end{array}$

AAGAAAACGC CAGGAAAAGG GCAGGGCGGC AGCCTGTCGC GTAACTTAGG ACTTGTGCGA 
$\begin{array}{llllll}8470 & 8480 & 8490 & 8500 & 8510 & 8520\end{array}$ CATGTCGTTT TCAGAAGACG GCTGCACTGA ACGTCAGAAG CCGACTGCAC TATAGCAGCG

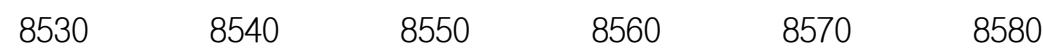
GAGGGGTTGG ATCAAAGTAC TTTGATCCCG AGGGGAACCC TGTGGTTGGC ATGCACATAC $\begin{array}{llllll}8590 & 8600 & 8610 & 8620 & 8630 & 8640\end{array}$ AAATGGACGA ACGGATAAAC CTTTTCACGC CCTTTTAAAT ATCCGTTATT CTAATAAACG $\begin{array}{llllll}8650 & 8660 & 8670 & 8680 & 8690 & 8700\end{array}$ CTCTTTTCTC TTAGGTTTAC CCGCCAATAT ATCCTGTCAA ACACTGATAG TTTAAACTGA $\begin{array}{llllll}8710 & 8720 & 8730 & 8740 & 8750 & 8760\end{array}$ AGGCGGGAAA CGACAATCTG ATCCAAGCTC AAGCTGCTCT AGCATTCGCC ATTCAGGCTG

$\begin{array}{llllll}8770 & 8780 & 8790 & 8800 & 8810 & 8820\end{array}$ CGCAACTGTT GGGAAGGGCG ATCGGTGCGG GCCTCTTCGC TATTACGCCA GCTGGCGAAA $\begin{array}{llllll}8830 & 8840 & 8850 & 8860 & 8870 & 8880\end{array}$ GGGGGATGTG CTGCAAGGCG ATTAAGTTGG GTAACGCCAG GGTTTTCCCA GTCACGACGT

$\begin{array}{llllll}8890 & 8900 & 8910 & 8920 & 8930 & 8940\end{array}$
TGTAAAACGA CGGCCAGTGC CAAGCTTGCA TGCGATCTTC AACCACCTGT GCTAGCTACT

$\begin{array}{llllll}8950 & 8960 & 8970 & 8980 & 8990 & 9000\end{array}$ CCACTGCTCC ATAGGCAATC ATCAATCAGT AATCCGTTCT GAAAAGAAGA TATAGGTGTG $\begin{array}{llllll}9010 & 9020 & 9030 & 9040 & 9050 & 9060\end{array}$ CGCAATCAGG AACGTTCTAG TTCGTGCTAG AAATCAGCAG CTCCTAAGTT AGCATCTCGA

$\begin{array}{llllll}9070 & 9080 & 9090 & 9100 & 9110 & 9120\end{array}$ TGAACTTAAA TGCTCGCTGC GGGCGTCCGG CGGAGATGAA GTTTGTGATA AACTTGGTCA $\begin{array}{llllll}9130 & 9140 & 9150 & 9160 & 9170 & 9180\end{array}$ TGACATTCAT ATATGTGCCT GGTGTACGGA GTAGTTCATC AGCAAACATA CACCTACTTC $\begin{array}{llllll}9190 & 9200 & 9210 & 9220 & 9230 & 9240\end{array}$ TACCTTATCC ATTTGGATTG CTCATGGCGG CTTTGATATG GAATTTGTAA TGAACTTGGT $\begin{array}{llllll}9250 & 9260 & 9270 & 9280 & 9290 & 9300\end{array}$ TATGACTTAT GACATACTGA TACTCGTAAC ATTCATAGAT ACTGACATAA ATTCATCAAC $\begin{array}{llllll}9310 & 9320 & 9330 & 9340 & 9350 & 9360\end{array}$ TACAATAGAT GAGATGGCTA GTCTTAGTAG AACAGTAGTC TCTCTTTCCG GCTTGCTCCA $\begin{array}{llllll}9370 & 9380 & 9390 & 9400 & 9410 & 9420\end{array}$ TTGGCTGATG ACGATGAACA ACTCGGACTC ATTGATTCCA GCATTATCTG ATTCTCGCAT 
$\begin{array}{llllll}9430 & 9440 & 9450 & 9460 & 9470 & 9480\end{array}$

TTCGAGGTCC GGATTAGGGT CTCACCGAGA TGTGGATAGA ATTGCCATGT CAGGAATTGA

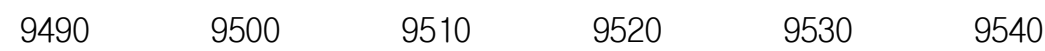
AGGAGGACGA GCCATATGTG CATATACATG ACGGGAGATC AAGCGGCCAG TCAAGAGGCT

$\begin{array}{llllll}9550 & 9560 & 9570 & 9580 & 9590 & 9600\end{array}$

AACTGCAACC CTATTATATA CGATCAGCCT GCTAGAACAC GTAGCACTGT CTTTTTTGTC

$\begin{array}{llllll}9610 & 9620 & 9630 & 9640 & 9650 & 9660\end{array}$

TGAACTCTGA AGATGAAAGG TTCAGAGAAA TGGCTCGCCT TATCCAAGCC GGCGATGGAT

$\begin{array}{llllll}9670 & 9680 & 9690 & 9700 & 9710 & 9720\end{array}$

GGAGGAGGAG GTAGCCGGCG CCCGCCTCAG GCAGTCGTCG CGATCACGCC GCCGCATCCC

$\begin{array}{llllll}9730 & 9740 & 9750 & 9760 & 9770 & 9780\end{array}$

GTCGCCTTGG AGACCGGGCC CCGACGCGGC CGACGCGGCG CCTACGTGGC CATGCTTTAT

$\begin{array}{llllll}9790 & 9800 & 9810 & 9820 & 9830 & 9840\end{array}$

TGCCTTATCC ATATCCACGC CATTTATTGT GGTCGTCTCT CCTGATCATT CTCATTCCCC

$\begin{array}{llllll}9850 & 9860 & 9870 & 9880 & 9890 & 9900\end{array}$

TGCCACGGTG ACCGTGCCCC CGGTGTTCTA TATATGCCCC CCGACGTCGA GGTCATTCGC

$\begin{array}{llllll}9910 & 9920 & 9930 & 9940 & 9950 & 9960\end{array}$

CACGAACACA TCGATCATCC ATCATCTACA AGAGATCGTC TAGAATTATT ACATCAAAAC

$\begin{array}{llllll}9970 & 9980 & 9990 & 10000 & 10010 & 10020\end{array}$ AAAAGATGAA GAATACCAGC TCGTTGTGTT TGCTTCTCCT CGTGGTGCTT TGCTCACTAA

$\begin{array}{llllll}10030 & 10040 & 10050 & 10060 & 10070 & 10080\end{array}$ CATGCAATTC GGGACAAGCA AATTCCGATT CCGAGTGTCC GCTCAGCCAC GACGGATACT

$\begin{array}{llllll}10090 & 10100 & 10110 & 10120 & 10130 & 10140\end{array}$ GCCTCCATGA TGGGGTCTGC ATGTACATTG AGGCCCTGGA CAAGTACGCG TGTAACTGCG

$\begin{array}{llllll}10150 & 10160 & 10170 & 10180 & 10190 & 10200\end{array}$ TGGTTGGCTA TATCGGCGAA AGGTGCCAGT ATCGGGACTT GAAGTGGTGG GAGCTTCGCT

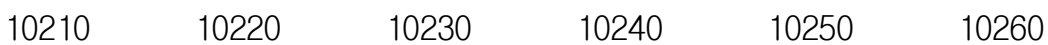

GATAGGTACC GAGCTCGGGC TCAAGCCCTA AACTGAACGG GATAGTCATG CTCAAACCAG

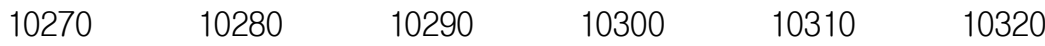
TTTCTACACG GCAAGAATTT ACTGATTCTT ATACTTTTGC AGTCAATTAA ATTATGGTTT

$\begin{array}{llllll}10330 & 10340 & 10350 & 10360 & 10370 & 10380\end{array}$ TTATATATGT AATTTTGTAT CCGATTGTAG CGTTCGAATA AGTAGGCAGG CTCTCTAGCC 


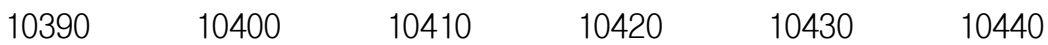

TCTAGGTTAA TTGCGGGGCA TATGTAGCTT GCCAGTTAAT TGTGTTTGTA TCACGCAGTT

$\begin{array}{llllll}10450 & 10460 & 10470 & 10480 & 10490 & 10500\end{array}$

TGTAACCGTT GGTGCAATAT ATAATGTCAG GTTCAGGATG CAGTAAAAAA TCATACTGCA

$\begin{array}{llllll}10510 & 10520 & 10530 & 10540 & 10550 & 10560\end{array}$

CCGATCAGTG AGTTTTTATG AATTC.... ......................

Supplementary Fig. 1. pJKS131 vector sequence to transfer the EGF to the rice genome. 